\title{
Polymixin-B hemoperfusion in a case intolerant to conventional antibiotic therapy
}

\author{
Gilnardo Novelli $^{1}$, Vincenzo Morabito ${ }^{1}$, Giancarlo Ferretti ${ }^{2}$, Simone Novelli ${ }^{3}$, Franco Ruberto ${ }^{4}$, \\ Milena Pernice $^{5}$, Cristina Donfrancesco ${ }^{5}$, Perluigi Benedetti Panici ${ }^{5}$ \\ ${ }^{1}$ Department "Paride Stefanini” General Surgery and Organs Transplant, Sapienza University, Rome, Italy \\ ${ }^{2}$ Department of Tropical and Infectous Disease, Sapienza University, Rome, Italy \\ ${ }^{3}$ Department of Clinical Engineering, Sapienza University, Rome, Italy \\ ${ }^{4}$ Department of Anesthesia and Intensive Care, Sapienza University, Rome, Italy \\ ${ }^{5}$ Department of Gynecology and Obstretics, Sapienza University, Rome, Italy \\ Email: novelligilnardo@virgilio.it
}

Received 18 January 2012; revised 10 February 2012; accepted 29 February 2012

\begin{abstract}
In this study we document how it was possible to resolve the septic complications of abdominal surgery, subjecting a patient severely allergic to intravenous antibiotics. We performed the hemoperfusion with polymyxin-B columns (PMX-DHP), guiding the therapy by assaying endotoxin activity and evaluating the clinical status of the patient after each session of PMX-DHP. The stabilization of hemodynamic and inflammatory frameworks were immediately observed after the PMXDHP. The patient could be safely discharged five days after the discontinuation of the therapy. Our findings confirms data already published in literature concerning the effectiveness of PMX-DHP.
\end{abstract}

Keywords: Sepsis; Endotoxin Activity; Antibiotics

\section{INTRODUCTION}

In this case study we report the particular case of a patient found to be severely allergic to two different pairs of antibiotics and therefore were forced to suspend double antibiotic therapy in course.

In recent years particular attention has been paid to antibiotic treatment coupled with standard therapy and carried out by hemoperfusion over columns with polymyxin-B, effective in neutralizing endotoxin originating from gramnegative bacteria [1].

Polymyxins have the unusual ability to bind to and neutralise endotoxin, this, rather than their antibacterial effects, might be of clinical value. The neutralisation of the lethal effects of endotoxin in animal models by polymyxin B has been known for more than 40 years but, their human clinical use has been limited due to nephrotoxicity and neurotoxicity. This limitation has been overcome by a mechanism which takes advantage of polymyxin without exposing the patient to its systemic effects. Polymyxin B-immobilized fiber hemoperfusion (PMX-DHP) is the only extracorporeal device reported to be safe and effective in septic patients [2-5].

The administration of this extracorporeal therapy could be usefully guided measuring endotoxin activity (EA) in whole blood [6,7] and using its value to initially enroll the patients and decide length of treatment [8].

In this case study we documented how it was possible to resolve the septic complications of abdominal surgery, subjecting a patient allergic to intravenous antibiotics, and hemoperfusion with polymyxin-B columns. Therapy was guided by assaying endotoxin activity and evaluating the clinical status of the patient after each session.

A Caucasian 51-year-old woman was admitted to our surgical ward to undergo total hysterectomy with bilateral salpingo-second Hudson-Dellepiane, omentectomy and peritoneal nodules were removed for bilateral papillary carcinoma of the ovary, fallopian tubes and nodular repetition. The clinical conditions were stable during the days following the surgery.

The patient maintained a drainage tube for four days. On the seventh day the patient showed a surgical wound dehiscence and during eighth day, fever $\left(38.8^{\circ} \mathrm{C}\right)$ associated with chills appeared. An increase of WBC (13* $\left.10^{3} / \mathrm{mm}^{3}\right)$, heart rate $(102$ beats $/ \mathrm{min})$ and a decrease of mean arterial pressure $(73 \mathrm{mmHg})$ were observed. An empirical antibiotic therapy was then started with quinolones [levofloxacin $1500 \mathrm{mg} / \mathrm{die}$ ] and third generation cephalosporins [ceftazidime $4 \mathrm{~g} / \mathrm{die}$ ], while urine and blood samples by peripherical venous access were sent for cultures.

On the ninth day, a toilet of the surgical wound was made without placing a drainage tube but at the same time antibiotic therapy was discontinued due to allergic and desquamative reactions. The patient presented an acute dermatitis erythematous pomfoide with initial evolution to- 
wards a Layer syndrome. Allergic reactions disappeared after discontinuation of antibiotics, but signs of sepsis persisted. During the tenth day the fever persisted $\left(39.1^{\circ} \mathrm{C}\right)$ and a new antibiotic therapy was prepared with aminoglycosides [gentamicin $160 \mathrm{mg} / \mathrm{die}$ ] and carbapenems [meropenem $2 \mathrm{~g} / \mathrm{die}]$. On the same day a CT scan excluded the presence of intra-abdominal abscesses.

At day 11 the patient showed a new desquamative allergic reaction to antibiotics that had to be suspended, but the fever persisted $\left(38.4^{\circ} \mathrm{C}\right)$ and the response of blood cultures showed the presence of an infection by Pseudomonas Aeruginosa. Moreover, since fever and chills appeared, a progressive reduction of daily diuresis (from $1460 \mathrm{ml} /$ day to $790 \mathrm{ml} /$ day within 2 days) and an increase of creatinine values (from $0.8 \mathrm{mg} / \mathrm{dl}$ to $1.4 \mathrm{mg} / \mathrm{dl}$ within 2 days) were observed. At day 12, the endotoxin activity was measured and resulted very high $(\mathrm{EA}>1.12)$ and we decided to perform the extracorporeal therapy with polymyxin-B (Day 0). During the following days the patient was treated for four days with only PMX-DHP until an EA value lower than 0.4 was obtained.

\section{METHODS}

Polymyxin B-hemoperfusion (PMX-DHP) [Toraymyxin ${ }^{\circledR}$, Toray Industries; Tokio; Japan] was performed using a column of polystyrene fibers to which polymyxin B (PMB) is covalently bonded. Each treatment was performed for two hours with a blood flow rate of $120 \mathrm{~mL} / \mathrm{min}$. The patient was treated with PMX-DHP every 24 hours until an $\mathrm{EA}<0.4$ was found.

EA in whole blood was measured as described by Romaschin et al. [9] using the EAA chemiluminescent assay (Spectra DiagnosticInc., Toronto, Ontario, Canada). Briefly, samples of EDTA anti-coagulated whole blood were incubated with saturating concentrations of antibody, then stimulated with zymosan.

The LPS/anti-LPS complex primes the patient's neutronphils for an augmented response.

The resulting respiratory burst activity was detected as light release using a chemiluminometer (SmartLine TL luminometer, Berthold); measuring basal (no antibody) and maximally stimulated ( $4600 \mathrm{pg} / \mathrm{mL}$ LPS plus antibody) responses in the same blood sample, the EA of the test specimen (antibody only) was then calculated. EA is expressed in relative units derived from the integral of the basal and stimulated chemiluminescent response.

Endotoxin activity can be classified as low for EA $<$ 0.4 , intermediate for $0.4 \leq \mathrm{EA}<0.6$ and high for $\mathrm{EA} \geq$ $0.6[6]$.

Vascular access was obtained by insertion of a double lumen dialysis catheter (Arrow International, Reading, PA, USA). The Sequential Organ Failure Assessment score was adopted for assessment of the severity before performing DHP-PMX. The EA Test was performed before and after PMX-DHP treatment. No antibiotic therapy was performed during PMX-DHP treatment.

\section{RESULTS AND DISCUSSIONS}

Immediately after the first PMX-DHP treatment, patients' temperature lowered to normal values. Table 1 reports both laboratory and clinical findings measured at enrollment and during the hemoperfusion therapy. Hemodynamics progressively resulted more stable after the fourth treatment: MAP increased, heart rate and lactates decreased.

Creatinine values and urinary output normalized. The inflammatory state decreased after the PMX-DHP therapy, i.e. the lowering of tumor necrosis factor (TNF)- $\alpha$ and interlukine(IL)- 6 values. The patients were discharged five days after the last PMX-DHP treatment.

In this report we documented a case of DHP-PMX therapy effective in treating a patient with postoperative abdominal sepsis, who was severely intolerant to different antibiotics administration. After the discontinuation due to intolerance of those two different antibiotic approaches, we decided to evaluate the endotoxin activity.

In presence of a very high value, we treated the patient with PMX-DHP. The EAA test can help us to determine after just 40 minutes the presence of a highly active endotoxin and leads us to determine the number of PMXDHP treatments to be carried out.

Table 1. Clinical and laboratory finding at enrolment and during the PMX-DHP therapy.

\begin{tabular}{cccccc}
\hline & Day 0 & Day 1 & Day 2 & Day 3 & Day 4 \\
\hline EA & 1.12 & 1.01 & 0.80 & 0.61 & 0.40 \\
mAP (mmHg) & 70 & 72 & 79 & 82 & 83 \\
HR (beats/min) & 106 & 93 & 83 & 77 & 74 \\
Lactate (mg/dl) & 3.7 & 3 & 2.5 & 1.9 & 1.5 \\
Hb (g/dL) & 8.9 & 8.8 & 9.1 & 9.3 & 9.3 \\
Platelets (10 $\left.\mathbf{3} / \mathbf{m m}^{3}\right)$ & 269 & 260 & 264 & 268 & 261 \\
Creatinine (mg/d) & 1.6 & 1.2 & 1.1 & 0.9 & 0.8 \\
Diuresis (mL/day) & 875 & 950 & 1050 & 1250 & 1750 \\
BUN (mg/dl) & 69 & 57 & 50 & 47 & 44 \\
WBC (10 $\left.\mathbf{3} / \mathbf{m m} \mathbf{3}^{3}\right)$ & 24 & 19.8 & 14 & 10 & 8.4 \\
Neutrophils (\%) & 94 & 90 & 84 & 72 & 64 \\
IL 6 (pg/ml) & 298 & 285 & 220 & 160 & 112 \\
TNF- $\boldsymbol{\alpha}$ (pg/ml) & 104 & 92 & 76 & 54 & 42 \\
SOFA score & 6 & 6 & 6 & 5 & 5 \\
\hline
\end{tabular}

Note: EA: endotoxin activity; mAP: mean arterial pressure; HR: heart rate; $\mathrm{Hb}$ : Hemoglobine; WBC: white blood cells; IL: Interlukine; TNF: tumor necrosis factor; SOFA: sequential organ failure assesment. 
The "dosing schedule" (number of PMX-DHP treatments) was determined by measuring the EA levels at 24 hours after each treatment. EA levels lowered after each treatment. In our clinical experience [8], we observed that percentage decrease was higher $(\sim 40 \%)$ for treatments starting from EA $\leq 0.7$ than those starting from EA $>0.9$ $(\sim 20 \%)$. In fact in this case, four treatments were necessary to determine a reduction of endotoxin activity $(\mathrm{EA}<0.4)$.

An improvement of organ dysfunction and the resolution of the septic state were observed. In this particular case, extracorporeal administration of polymyxin-B was the only antibiotic therapy tolerated by the patient, thus confirming the absence of side effects associated with PMX-DHP, as already known from the literature.

There are still some issues to be discussed regarding the resolution of the infection status and the related septic state. First, despite the very short-term systemic antibiotic therapy which could have had minimal effective on infection, especially in the presence of a low bacterial colonization.

In this case, the only cause of sepsis could be the endotoxin released from lysis of cell membranes and/or translocated directly from the gastro-intestinal tract; the polymyxin-B column was therefore effective in removing those endotoxins.

Second, two works on animals and in-vitro [10,11] reported a residual antimicrobial activity of polymyxin-B in the column; thus we might assume that the sequence of four antimicrobial adjuvant treatment resulted in the elimination of infection and its bacterial products.

In this report, an allergy to antibiotics manifested by the patient led to the sole use of extracorporeal therapy. The effective removal of endotoxins resulted in a resolution of the septic state.

\section{REFERENCES}

[1] Tani, T., Shoji, H., Guadagni, G. and Perego, A. (2010) Extracorporeal removal of endotoxin: The polymyxin B-immobilized fiber cartridge. Contributions to Nephrology, 167, 35-44. doi:10.1159/000315917

[2] Cruz, D.N., Perazella, M.A., Bellomo, R., et al. (2007)
Effectiveness of polymyxin B-immobilized fiber column in sepsis: A systematic review. Critical Care, 11, R47. doi:10.1186/cc5780

[3] Cantaluppi, V., Assenzio, B., Pasero, D., et al. (2008) Polymyxin-B hemoperfusion inactivates circulating proapoptotic factors. Intensive Care Medicine, 34, 1638-1645. doi:10.1007/s00134-008-1124-6

[4] Shoji, H. (2003) Extracorporeal endotoxin removal for the treatment of sepsis: Endotoxin adsorption cartridge (Toraymyxin). Therapeutic Apheresis and Dialysis, 7, 108114. doi:10.1046/j.1526-0968.2003.00005.x

[5] Shimizu, T., Yoshihiro, E., Tsuchihashi, H., et al. (2006) Endotoxin apheresis for sepsis. Transfusion and Apheresis Science, 35, 271-282. doi:10.1016/j.transci.2006.06.006

[6] Marshall, J.C., Foster, D., Vincent, J.L., et al. (2004) Diagnostic and prognostic implications of endotoxemia in critical illness: Results of the MEDIC study. Journal of Infectious Diseases, 190, 527-534. doi:10.1086/422254

[7] Monti, G., Bottiroli, M., Pizzilli, G., et al. (2010) Endotoxin activity level and septic shock: A possible role for specific anti-endotoxin therapy? Contributions to $\mathrm{Ne}$ phrology, 167, 102-110. doi:10.1159/000315924

[8] Novelli, G., Ferretti, G., Ruberto, F., et al. (2010) Early management of endotoxemia using the endotoxin activity assay and polymyxin B-based hemoperfusion. Contributions to Nephrology, 167, 91-101. doi:10.1159/000315923

[9] Romaschin, A.D., Harris, D.M., Ribeiro, M.B., et al. (1998) A rapid assay of endotoxin in whole blood using autologous neutrophil-dependent chemiluminescence. Journal of Immunological Methods, 212, 16-85. doi:10.1016/S0022-1759(98)00003-9

[10] Hanasawa, K., Tani, T. and Kodama, M. (1989) New approach to endotoxic and septic shock by means of polymyxin B immobilized fiber. Surgery Gynecology \& Obstetrics, 168, 323-331.

[11] Shoji, H., Tani, T., Hanasawa, K. and Kodama, M. (1998) Extracorporeal endotoxin removal by polymyxin B immobilized fiber cartridge: Designing and antiendotoxin efficacy in the clinical application. Therapeutic Apheresis, 2, 3-12. doi:10.1111/j.1744-9987.1998.tb00066.x 